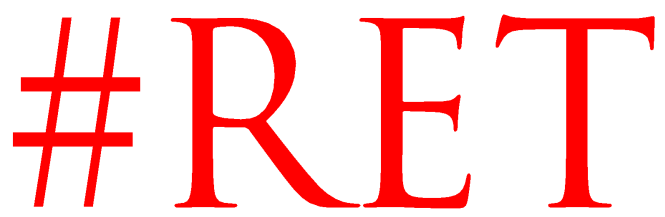

Revista Española de la Transparencia

Núm. 10. Primer Semestre 2020

ISSN 2444-2607. Págs. 49-64

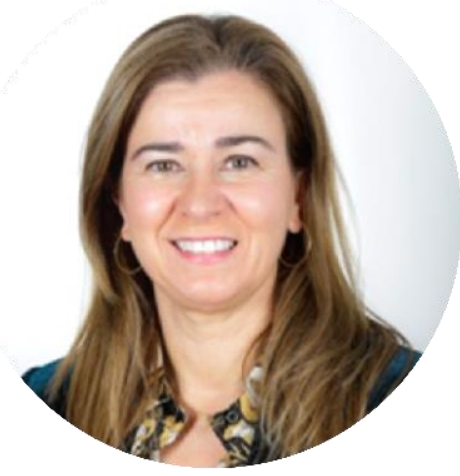

$M^{\text {a }}$ Teresa Ruiz-Sillero Bernal 1

Universidad de Cádiz. España

\title{
El primer expediente sancionador incoado por el incumplimiento de la Ley de Transparencia Pública de Andalucía
}

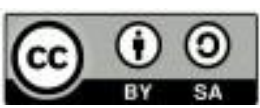

RECIBIDO: 24 de octubre de 2019 ACEPTADO: 30 de enero de 2020

RESUMEN: El Consejo de Transparencia y Protección de Datos de Andalucía, conforme a sus competencias, instó la incoación de un expediente sancionador por el incumplimiento de la Ley 1/2014, de 24 de junio, de Transparencia Pública de Andalucia. Se explicará el procedimiento regulado en el Título VI de dicha norma, por el cual se instó dicha incoación tras la resolución en la que se declaró el incumplimiento de la obligación de publicidad activa del gobierno de la Junta de Andalucia, según la denuncia interpuesta por un grupo político con representación en el Parlamento de Andalucía. Así mismo, se analizará la tramitación de dicho expediente sancionador por parte de la Junta de Andalucía.

PALABRAS CLAVE: Transparencia, incumplimiento, expediente sancionador, sanción.

CONTENIDOS: 1. Introducción. - 2. La denuncia ante el Consejo de Transparencia y Protección de Datos de Andalucía. - 3. La resolución de declaración de incumplimiento de la Ley de Transparencia Pública de Andalucia. - 4. La resolución del Consejo de Transparencia y Protección de Datos de Andalucia por la que se insta incoar un expediente sancionador. - 5. La incoación del expediente sancionador por parte de la Consejeria de Hacienda y Administración Pública de la Junta de Andalucía. - 6. Conclusiones. - Bibliografía.

\footnotetext{
${ }^{1}$ Licenciada en Derecho por la Universidad de Sevilla. Especialidad en Derecho Público. Abogada y Gestor Administrativo. Alumna de doctorado de la Universidad de Cádiz, Programa de Derecho.
} 


\section{$M^{a}$ Teresa Ruiz-Sillero Bernal}

\section{The first administrative prosecution file initiated by breach of the Andalusia public transparency law}

ABSTRACT: The Council of Transparency an Data Protection of Andalusia, in accordance with its powers, urged the opening of a sanction file for breach of Law 1/2014, of June 24, on Public Transparency of Andalusia. The procedure regulated in Title VI of said norm will be explained, by which said initation was urged after the resolution in which the breach of the obligation of active publicity of the Government of the Junta de Andalucia was declared, according to the complaint filed by a political group with representation in the Parliament of Andalusia. Likewise, the processing of said disciplinary proceedings by the Junta de Andalucia will be analized.

KEYWORDS: Transparency, non-compliance, disciplinary proceedings, sanction.

\section{INTRODUCCIÓN.}

Con posterioridad a la Ley estatal de Transparencia, Acceso a la Información Pública y Buen Gobierno, Ley 19/2013, de 9 de diciembre, (LTBG, en adelante) se aprobó por la Comunidad Autónoma de Andalucia la Ley 1/2014, de Transparencia Pública de Andalucía de 24 de junio, (LTPA, en adelante). Una de las grandes aportaciones de esta norma autonómica es la inclusión de un régimen sancionador, establecido en el Título VI, que regula el procedimiento, la competencia sancionadora, los responsables y las infracciones y sanciones. Tras la norma andaluza se aprobarian otras normas autonómicas que también incluyen un régimen sancionador: En la Región de Murcia, la Ley 12/2014, de 16 de diciembre; en Canarias, la Ley 12/2014, de 26 de diciembre; en Cataluña, la Ley 19/2014, de 29 de diciembre; en Castilla y León, la Ley 3/2015, de 4 de marzo; en la Comunidad Valenciana, la Ley 2/2015, de 2 de abril; y en Castilla-La Mancha, la Ley 4/2016, de 15 de diciembre.

En este artículo se analiza detalladamente el primer caso en el que el Consejo de Transparencia y Protección de Datos de Andalucia (CTPDA, en adelante) instó la incoación de un expediente sancionador a la Consejería de Hacienda y Administración Pública de la Junta de Andalucía por el incumplimiento de las obligaciones de publicidad activa de la Ley de Transparencia Pública de Andalucia.

Se abordará la interposición de la denuncia ante el CTPDA, la resolución de dicho Consejo que instó la incoación del expediente sancionador, la tramitación de dicho expediente sancionador y sus conclusiones. Se pretende analizar la aplicación del régimen sancionador que recoge la LTPA. Así mismo, se abordará para este caso 


\section{El primer expediente sancionador ...}

concreto tanto la problemática de la competencia que se le reconoce al CTPDA para instar la incoación del expediente sancionador como la dificultad de la imposición de una sanción en la materia.

\section{LA DENUNCIA ANTE EL CONSEJO DE TRANSPARENCIA Y PROTECCIÓN DE DATOS DE ANDALUCÍA}

Conforme al artículo 23 de la LTPA, el CTPDA asume unas competencias en materia de control de transparencia pudiendo efectuar "requerimientos para la subsanación de los incumplimientos que pudieran producirse de las obligaciones establecidas en este título (el Título II sobre la publicidad activa)". Dicho Consejo podrá actuar por iniciativa propia o como consecuencia de denuncia lo que, según señala Falcón (2018: 496), otorga a la ciudadanía la posibilidad de participar en la vigilancia y control del cumplimiento de las obligaciones de publicidad activa por parte de los sujetos obligados y añade que así, la LTPA incorpora en relación con el control de la publicidad activa dos elementos relevantes: la denuncia por parte de la ciudadanía y la posibilidad de realizar requerimientos por parte del Consejo para subsanar incumplimientos, ya sea por propia iniciativa o como consecuencia de las mencionadas denuncias.

El mencionado Falcón (2018: 499) analiza el procedimiento para las denuncias ante el Consejo y explica que las mismas se pueden presentar cumplimentando los formularios diseñados al efecto, que pueden ser tramitados de forma telemática a través de la "Ventanilla Electrónica", o también mediante la remisión de la denuncia, donde conste la identidad del denunciante, la entidad denunciada y el motivo de la denuncia a la sede del Consejo.

Basándose en el citado artículo 23 de la LTPA el grupo Parlamentario del Partido Popular en el Parlamento de Andalucía presentó una denuncia ante el CTPDA el 28 de octubre de 2016, la cual fue tramitada con el número de expediente 59/2016. En la referida denuncia se pusieron de manifiesto unos hechos que suponían, según el denunciante, el incumplimiento de las obligaciones de publicidad activa por parte de la Consejería de Hacienda y Administración Pública de la Junta de Andalucía por vulneración del artículo 13.1.b) de la LTPA, sobre información de relevancia juridica.

Los hechos concretos denunciados fueron la no publicación en el Portal de Transparencia del Anteproyecto de la Ley de Presupuesto de la Comunidad Autónoma de Andalucía para el año 2017 en el momento en que el Consejo de Gobierno de la Junta de Andalucía remitió al Consejo Consultivo de Andalucía dicho anteproyecto para su dictamen, es decir, el 18 de octubre de 2016. El Consejo Consultivo de Andalucía emitió dictamen ${ }^{2}$ el día 24 de octubre del citado año. A la

\footnotetext{
2 Dictamen del Consejo Consultivo de Andalucía número 0672/2016, de fecha 24 de octubre de 2016, número de marginal I.26. https://server.knosys.es/ccandalucia/knpag?x=index\#
} 


\section{$M^{a}$ Teresa Ruiz-Sillero Bernal}

fecha de la denuncia, el 28 de octubre, seguía sin haberse publicado dicho anteproyecto de Ley en el Portal de Transparencia de la Junta de Andalucía.

Habria que recordar aquí la obligación que tienen las administraciones públicas andaluzas de publicar información de relevancia jurídica conforme al artículo 13.1.b) de la LTPA refiriéndose dicho precepto a:

"Los anteproyectos de ley cuando, tras la preceptiva elevación por la Consejería competente, sean conocidos por el Consejo de Gobierno. Asimismo, los anteproyectos de ley y los proyectos de decretos legislativos se publicarán cuando se soliciten los dictámenes, en su caso, al Consejo Económico y Social de Andalucía y al Consejo Consultivo de Andalucía. Y, finalmente, los proyectos de ley tras su aprobación por el Consejo de Gobierno".

En la denuncia se solicitaba en primer lugar que:

"Se requiera tanto al Consejo de Gobierno de la Junta de Andalucía como a la Consejería de Hacienda y Administración Pública para que, en primer lugar, publique con carácter urgente y en el día de hoy tanto el Anteproyecto de Ley de Presupuesto de la Comunidad Autónoma de Andalucía para el año 2017 en el Portal de la Transparencia de la Administración de la Junta de Andalucía como el Proyecto de Ley de Presupuesto de la Comunidad Autónoma de Andalucia para el año 2017 tras su aprobación por el Consejo de Gobierno; y para que, en segundo lugar, se adopten las medidas oportunas por ese Consejo de Transparencia y Protección de Datos de Andalucia para evitar que en posteriores ocasiones no se publiquen los anteproyectos de ley en general, y los anteproyectos de Ley del Presupuesto de la Comunidad Autónoma de Andalucía, en particular."

Y en segundo lugar se solicitaba que:

"Conforme al régimen sancionador regulado en el Título VI de la Ley 1/2014, de 24 de junio, inste la incoación del procedimiento previsto en el artículo 57 de dicho texto legal al objeto de fijar los responsables, las infracciones y en su caso, las sanciones oportunas por el incumplimiento reiterado del artículo 13.1.b) de la Ley de Transparencia Pública de Andalucia."

La denuncia presentada ponía el foco en la tramitación de una ley que es la más importante de cuantas se tramitan cada año en el Parlamento de Andalucía, la Ley de Presupuesto para la Comunidad Autónoma. No se escapa el interés político de conocer el contenido del Anteproyecto de la misma en cuanto ello fuera legalmente posible, como un modo de control al Gobierno y toda vez que éste procedia a explicar el contenido del anteproyecto de Ley sin que la oposición dispusiera de información sobre el mismo. Y tal y como señala Fernández Ramos 


\section{El primer expediente sancionador ...}

(2015: 68) debe entenderse que el mandato de publicidad activa no se ciñe a los contenidos informativos explicitados en el Título II de la Ley, sino que se extiende a todas aquellas informaciones relacionadas con el funcionamiento y control de la actuación pública relevantes para garantizar la transparencia de su actividad.

Tal y como detalla Falcón (2018: 496) otro elemento diferenciador de la normativa andaluza de transparencia es el establecimiento de un régimen sancionador específico, que no se incluye en la legislación básica. Este régimen sancionador se describe en el Título VI LTPA. En lo que se refiere especificamente al incumplimiento de obligaciones de publicidad activa, se consideran las siguientes infracciones: de carácter disciplinario, imputables a las autoridades, directivos y el personal al servicio de las entidades previstas en el artículo 3 de la LTPA (artículo 52 LTPA) y las imputables a las entidades de naturaleza privada a las que se refiere el artículo 5 LTPA (artículo 54 LTPA). Las sanciones correspondientes a las infracciones mencionadas se establecen en los artículos 55 y 56 de la LTPA, distinguiendo así mismo entre sanciones disciplinarias y sanciones a otras entidades con multas pecuniarias.

Frente al prácticamente inexistente régimen sancionador en materia de transparencia en la Ley estatal, según la guía de Wolters Kluwer en Actualidad Administrativa3, la LTPA en su artículo 52 hace extensiva la condición de personas responsables de las infracciones a quienes, aun a título de simple inobservancia, realicen acciones o incurran en las omisiones tipificadas en la ley con dolo, culpa o negligencia. Y detalla que las infracciones tipificadas se distinguen en función de la materia: de carácter disciplinario y en materia de publicidad activa; incluso prevé la comisión de infracciones por las entidades de naturaleza privada (lo que no se da en este caso concreto).

\section{LA RESOLUCIÓN DE DECLARACIÓN DE INCUMPLIMIENTO DE LA LEY DE TRANSPARENCIA PÚBLICA DE ANDALUCÍA}

El CTPDA, una vez recibida la denuncia analizada en el punto anterior, concedió a la Consejería de Hacienda y Administración Pública un plazo de 10 días al objeto de que formulara las alegaciones que estimara oportunas y aportara los documentos y justificaciones que considerara pertinentes.

La Consejería denunciada alegó, entre otras cuestiones para justificar la no publicación del Anteproyecto de Ley de Presupuestos, la especificidad y celeridad con la que se tramita, teniendo reconocido un carácter especial por lo que está previsto una tramitación también especial y diferenciada del resto de las normas legales. Además, añadió, que muchas de las cifras y la cuantificación de ingresos y gastos que se incluyeron en el texto final no acompañaban inicialmente al borrador

\footnotetext{
3 Wolters Kluwer 2019. Régimen sancionador en materia de transparencia, Actualidad Administrativa, número 10.
} 


\section{$M^{a}$ Teresa Ruiz-Sillero Bernal}

que se sometió a dictamen por imposibilidad técnica en tanto aún no había culminado el debate en el seno del Consejo de Gobierno. También alegó, respecto a la petición de instar la incoación de un expediente sancionador, que "predicar de un incumplimiento merecedor de sanción por no publicar una materia en un día exacto cuando la exigencia es actualizar y publicar trimestralmente es cuanto menos exagerada y alarmista".

A la vista de las alegaciones presentadas, el CTPDA dictó la Resolución número PA-3/2017, de 11 de enero ${ }^{4}$, a favor del denunciante, en la que resolvió:

"Primero. Declarar que la Consejería de Hacienda y Administración Pública ha incumplido la obligación de publicidad activa establecida en el artículo 13.1.b) de la Ley 1/2014, de 24 de junio, de Transparencia Pública de Andalucia.

Segundo. Requerir expresamente a la Consejería de Hacienda y Administración Pública para que, en lo sucesivo, proceda a la publicación de los Anteproyectos de Ley del Presupuesto de la Comunidad Autónoma de Andalucía en el Portal de Transparencia en el momento en que se solicite el dictamen al Consejo Consultivo".

Reseñar, tal y como consta en el fundamento juridico primero de la referida Resolución, que es el director del CTPDA quien tiene la competencia para la resolución de las denuncias presentadas de acuerdo a lo previsto en el artículo 10.3.b) de los Estatutos del CTPDA ${ }^{5}$ y en el artículo 48.1.g) de la LTPA, en conexión con el artículo 23 del mismo texto legal.

En el fundamento jurídico segundo de la mencionada Resolución se señalaba expresamente que:

"La aplicación de lo dispuesto en el artículo 13.1 b) de la LTPA al presente caso conduce directamente a concluir que el mismo día desde que fue solicitado dicho dictamen (al Consejo Consultivo de Andalucía) debió haberse publicado el Anteproyecto de Ley del Presupuesto en el citado Portal de Transparencia".

Y se añadia en el fundamento jurídico tercero, después de reconocer las singularidades procedimentales y técnicas alegadas por la Viceconsejería de Hacienda y Administración Pública, que:

\footnotetext{
4 Resolución número PA-3/2017, de 11 de enero, del Consejo de Transparencia y Protección de Datos de Andalucia https://www.ctpdandalucia.es/sites/default/files/denuncias/res-pa-03-2017.pdf

5 Decreto 434/2015, de 29 de septiembre, por el que se aprueban los Estatutos del Consejo de Transparencia y Protección de Datos de Andalucía https://www.juntadeandalucia.es/boja/2015/193/1
} 


\section{El primer expediente sancionador ...}

"Es evidente que nada impedia elevar al Portal de Transparencia de la Junta de Andalucía el mismo texto que fue remitido al Consejo Consultivo a fin de que emitiera su dictamen".

Por último, en el fundamento jurídico cuarto, en contestación a una de las alegaciones presentadas por la Viceconsejería de Hacienda y Administración Pública, se afirma que "de la referida posibilidad de prescindir de la consulta pública no cabía extraer ninguna consecuencia relevante para la denuncia presentada". $Y$ en el fundamento jurídico quinto no estima la petición del denunciante para que se publique con carácter urgente el Anteproyecto de la Ley de Presupuesto de la Comunidad Autónoma para el año 2017 en el Portal de la Transparencia "habida cuenta de que en el curso de la tramitación de esta denuncia se procedió a la publicación del reiterado Proyecto y el Parlamento ha llegado a probar la Ley 10/2016, de 27 de diciembre, del Presupuesto de la Comunidad Autónoma para el año 2017" por lo que añade, "sólo cabe requerir el cumplimiento ad futurum de la obligación de publicidad activa objeto de la denuncia".

La particularidad de la ya mencionada resolución número PA-3/2017, de 11 de enero, radica en que el director del CTPDA no se pronunció sobre el segundo petitum de la denuncia. No hubo un pronunciamiento sobre la solicitud de que instase la incoación del procedimiento previsto en el artículo 57 de la LTPA al objeto de fijar los responsables, las infracciones y en su caso, las sanciones oportunas por el incumplimiento reiterado del artículo 13.1.b) de la LTPA. Sin embargo, en el hecho primero de la referida resolución sí se hizo referencia a esa solicitud del denunciante. Ello no se correspondería con el principio de congruencia de las resoluciones.

El artículo 88.1 de la Ley 39/2015, de 1 de octubre, del Procedimiento Administrativo Común de las Administraciones Públicas (en adelante LPAC) establece que "la resolución que ponga fin al procedimiento decidirá todas las cuestiones planteadas por los interesados y aquellas otras derivadas del mismo". En este sentido, García de Enterría y Fernández (2017: 536) consideran que de acuerdo a los artículos 88 y 119 LPAC "la resolución de todo procedimiento administrativo debe ser, por supuesto, congruente con las peticiones formuladas por los interesados, en el doble sentido de que sus alegatos deben ser tenidos en cuenta por ella "sin perjuicio de que los acoja o los rechace, según legalmente corresponda" (S. de 7 de diciembre de 1972), y de que las concretas pretensiones que hayan sido ejercitadas deben ser objeto de pronunciamiento pertinente para no causarles indefensión (Sentencias de 3 de diciembre de 1993 y 3 de noviembre de 1998), pronunciamiento que debe guardar en todo caso la debida correspondencia con aquellas (S. de 16 de enero de 1996), so pena de nulidad."

Ante esta falta de pronunciamiento, el Grupo Parlamentario Popular en el Parlamento de Andalucía interpuso un recurso potestativo de reposición ante el 


\section{$M^{a}$ Teresa Ruiz-Sillero Bernal}

CTPDA con fecha 27 de enero de 2017, el cual se analizará en el siguiente apartado. Conforme al pie de recurso de la citada resolución, la misma ponía fin a la vía administrativa y se podía interponer recurso potestativo de reposición ante el CTPDA o interponer recurso contencioso-administrativo ante el Juzgado de lo Contencioso-Administrativo de Sevilla.

\section{LA RESOLUCIÓN DEL CONSEJO DE TRANSPARENCIA Y PROTECCIÓN DE DATOS DE ANDALUCIAA POR LA QUE SE INSTA INCOAR UN EXPEDIENTE SANCIONADOR.}

Una vez presentado el recurso potestativo de reposición, el CTPDA dio traslado del mismo a la Consejeria de Hacienda y Administración Pública. Conforme a lo previsto en el artículo 118.2 de la Ley 39/2015, de 1 de octubre, del Procedimiento Administrativo Común de las Administraciones Públicas le fue concedido a dicha Consejería un plazo de alegaciones de diez días.

La Consejería de Hacienda y Administración Pública en sus alegaciones fechadas el 14 de febrero de 2017, planteó que el CTPDA no tenía un especial deber de motivar su decisión de no atender la pretensión de la recurrente y, además, argumentaba que:

"Para que proceda la exigencia de responsabilidad en el ámbito administrativo deben concurrir una serie de requisitos predeterminados normativamente pues la reacción punitiva de la Administración queda reservada a incumplimientos especialmente gravosos, no bastando con la existencia de meras irregularidades para que surja el reproche administrativo". Y añadía que "aun cuando con frecuencia se declaran incumplimientos de la normativa sobre transparencia esos incumplimientos no llevan aparejada la instancia para el inicio de procedimiento sancionador".

A la vista de las alegaciones presentadas y en contestación al recurso de reposición presentado por el Grupo Parlamentario Popular en el Parlamento de Andalucía, el CTPDA emitió una resolución el 24 de febrero de 2017 en la que declaró la terminación del procedimiento del recurso de reposición por pérdida sobrevenida del objeto del recurso, sin entrar a valorar, por tanto, las alegaciones presentadas por la Consejería de Hacienda y Administración Pública. Y es que tres días antes había emitido una resolución por la que instaba a la Consejería de Hacienda y Administración Pública para que incoara el procedimiento correspondiente por incumplimiento de obligación de publicidad activa. Tenemos ya, por tanto, la primera resolución del CTPDA en la que se insta a incoar un expediente sancionador por el incumplimiento de la LTPA. En dicha resolución, de fecha 21 de febrero de 2017, se acordó:

"Primero. Instar a la Consejería de Hacienda y Administración Pública para que incoe el procedimiento correspondiente por incumplimiento de 


\section{El primer expediente sancionador ...}

obligación de publicidad activa de la Ley 1/2014, de 24 de junio, de Transparencia Pública de Andalucia, acordado en la Resolución PA-372017.

Segundo. Conforme lo previsto en el artículo 57.2 LTPA, la Consejería de Hacienda y Administración Pública vendrá obligada a comunicar a este Consejo el resultado del mismo."

Resulta de interés analizar los fundamentos juridicos de la Resolución de 21 de febrero de 2017 del CTPDA. En el fundamento jurídico primero consta que corresponde al director de dicho Consejo, conforme a lo dispuesto en el artículo 48.1.h) la competencia para "instar la incoación de expedientes disciplinarios o sancionadores, de acuerdo a las previsiones del Título VI (de la LTPA)". En este sentido, Rueda Gómez (2015: 263) señala que esta potestad de control es, sin duda, la más relevante dado que otorga al CTPDA la capacidad de dictar resoluciones con fuerza vinculante.

En el fundamento jurídico segundo argumenta que "constatado el incumplimiento de la LTPA y una vez que la Resolución PA-3/2017 de 11 de enero ha adquirido firmeza en vía administrativa procede, en virtud de lo dispuesto en el artículo 57.2 de la LTPA, instar al órgano denunciado a incoar el procedimiento correspondiente, el cual resultará obligado a su incoación y a comunicar al Consejo el resultado del mismo." A este respecto, Pérez Monguió (2015: 319) llama a la atención sobre la facultad que se atribuye al director del Consejo de la Transparencia y Protección de Datos de Andalucía, siguiendo el ejemplo del artículo 38.2.e) de la LTBG de "instar la incoación de expedientes disciplinarios o sancionadores de acuerdo al Título VI" [art. 48.1.b)]. En el caso de la LTBG, el órgano competente deberá motivar, en su caso, su decisión de no hacerlo, pero solo corresponde a este último la facultad de decidir si lo incoa o no. La LTPA ha dado un paso más cuando dispone que "el órgano competente estará obligado a incoar el procedimiento y a comunicar al Consejo el resultado del mismo" (art. 57.2). Por tanto, añade el autor, los órganos competentes están, por la LTPA, obligados a incoar el procedimiento, a diferencia de la LTBG que exclusivamente requiere que, en caso de que no se incoe, se motive.

El CTPDA quiso, además, en el fundamento jurídico segundo aclarar que al carecer de competencia sancionadora no le correspondía determinar la responsabilidad derivada de dicho incumplimiento sino ceñirse a instar, conforme al artículo 57 de la LTPA, que ese procedimiento sea incoado por el órgano competente y que sería en el procedimiento que se incoara e instruyera donde se determinarían, en su caso, dichas responsabilidades.

La referida Resolución de 21 de febrero de 2017 del CTPDA ponía fin a la vía administrativa $y$, por tanto, podía interponerse recurso potestativo de reposición ante el CTPDA o interponer directamente recurso contencioso-administrativo ante el Juzgado de lo Contencioso-Administrativo de Sevilla. La Viceconsejería de 


\section{Ma Teresa Ruiz-Sillero Bernal}

Hacienda y Administración Pública optó por interponer con fecha 30 de marzo de 2017, un recurso potestativo de reposición ante el CTPDA.

La Viceconsejería en su recurso alegó que la citada resolución era nula de pleno derecho en virtud de lo previsto en el artículo 47.1. letras a) y e) de la LPAC, al haberse dictado extemporáneamente fuera del procedimiento legalmente previsto para ello. Consideraba así mismo que se había denegado tácitamente la petición de incoación de expediente sancionador formulada por el denunciante. Y añadía que:

"La Consejería actúa de buena fe y no recurre la Resolución de 11 de enero (que declaró el incumplimiento) en la medida que el contenido obligacional que de la misma se deriva se concreta en fijar, "en lo sucesivo", el momento procedimental en el que debería procederse a la publicación de los anteproyectos de leyes de presupuestos en el Portal de la Transparencia. Por tanto, se conculca de manera flagrante el derecho de defensa de esta Administración, sin que se pueda mediante una resolución posterior y de forma sorpresiva, instarse la incoación de un procedimiento en base a la firmeza de una resolución anterior, en la que no se derivaba exigencia alguna de responsabilidad por la declaración que la misma se contiene. Y añade que, en consecuencia, para que sea procedente la exigencia de responsabilidad administrativa o disciplinaria es necesario que concurra el incumplimiento una voluntad cualificada que permita calificar la conducta como dolosa culposa o negligente. Por tanto, no todo incumplimiento de la normativa sobre transparencia pública genera una responsabilidad administrativa disciplinaria, exigiéndose ese elemento subjetivo para que sea procedente el reproche en esos ámbitos. En el presente caso, la actuación de esta consejería no puede calificarse como dolosa, culposa o negligente."

Además, la Consejería solicitó la suspensión de la resolución recurrida, al fundamentarse el recurso en las causas de nulidad de pleno derecho y alegando que su inmediata ejecutividad causaria un grave perjuicio a la imagen institucional de la Consejería en la que decía, existía un impulso decisivo por la implantación efectiva de la transparencia no causando, además, la suspensión solicitada daños a terceros.

Presentado este recurso de reposición el director del CTPDA emite un Acuerdo de 25 de abril de 2017, fundamentando dicha decisión en el artículo 23.1 de la LPAC 6 .

\footnotetext{
6 Artículo 23.1 de la LPAC: "Excepcionalmente, cuando se hayan agotado los medios personales y materiales disponibles a los que se refiere el apartado 5 del artículo 21, el órgano competente para resolver, a propuesta, en su caso, del órgano instructor o el superior jerárquico del órgano competente para resolver, podrá acordar de manera motivada la ampliación del plazo máximo de resolución y notificación, no pudiendo ser este superior al establecido para la tramitación del procedimiento".
} 


\section{El primer expediente sancionador ...}

por el que se ampliaba en un mes el plazo máximo de resolución y notificación del citado recurso de reposición. Argumentaba la falta de medios personales que le imposibilitaba poder cumplir los plazos previstos en la normativa de aplicación y lanzaba una llamada de atención haciendo constar que "la aprobación de la Relación de Puestos de Trabajo del Consejo, que incluye la dotación mínima de plazas para el ejercicio de sus competencias, ha de poner fin a esta situación de precariedad de recursos humanos en el Área de Transparencia. La situación descrita impide, a pesar de los esfuerzos del personal asignado, poder cumplir los plazos previstos en la normativa de aplicación".

Seguidamente, el CTPDA concedió al Grupo Parlamentario Popular un plazo de alegaciones para que formulase lo que estimase conveniente respecto del recurso de reposición presentado por la Viceconsejería. Aquel se opuso a la suspensión argumentando, entre otros motivos, que resultaba jurídicamente irrelevante el posible grave perjuicio a la imagen institucional de la Consejería alegado por ésta y que no era el momento procedimental para apreciar si concurrían o no los elementos para que fuera punible una acción.

Finalmente, el CTPDA emite una Resolución de fecha 13 de junio de 2017 en la que desestima tanto el recurso de reposición interpuesto por la Sra. Viceconsejera de Hacienda y Administración Pública, contra la Resolución de 21 de febrero de 2017. por la que se instaba la incoación de procedimiento por incumplimiento de la LTPA como la solicitud de suspensión de ejecución de la misma. Resulta interesante destacar los siguientes fundamentos jurídicos de dicha Resolución:

En el fundamento jurídico tercero se niega la nulidad de pleno derecho de la Resolución de 21 de febrero de 2017 por haberse otorgado a la Consejería de Hacienda y Administración Pública los correspondientes plazos de alegaciones.

En el fundamento jurídico cuarto se recalca que el CTPD al instar la incoación del expediente sancionador "no ha hecho, lisa y llanamente, más que cumplir con lo dispuesto en la LTPA". Señala que una vez comprobado el incumplimiento de la obligación de publicidad activa al Consejo no le queda otra alternativa que llevar a afecto lo que impone en términos inequivocos el artículo 57.2 de la LTPA. Y añade el CTPDA que las funciones que se le atribuyen respecto al régimen sancionador llegan justamente hasta aqui, por consiguiente, "so pena de rebasar nuestro ámbito competencial acotado por el Legislador, ninguna decisión podemos adoptar sobre la determinación de la responsabilidad que eventualmente pueda derivarse de la inobservancia del artículo 13.1.b) de la LTPA; tarea ésta que corresponde al órgano que ostente la competencia sancionadora según lo previsto en el artículo 58.1 LTPA."

$Y$ en el fundamento jurídico sexto se indica que no se ha argumentado específicamente por parte de la Viceconsejería de Hacienda y Administración Pública qué perjuicios de dificil o imposible reparación concurrían más allá de la genérica e inconcreta alegación relativa a la erosión de la imagen institucional 


\section{$M^{a}$ Teresa Ruiz-Sillero Bernal}

derivada de la ejecución, por lo que no cabria estimar la suspensión de la ejecución de la Resolución recurrida dado que también se rechazó de plano la concurrencia de las causas de nulidad invocadas.

Cabría, por último, en este apartado, plantear dos cuestiones en relación a la Resolución del CTPDA de 21 de febrero de 2017 por la que acordó, por primera vez, instar la incoación de un expediente sancionador por incumplimiento de la LTPA.

En primer lugar, si conforme al artículo 57.2 de la LTPA el CTPDA debe actuar siempre instando la incoación del procedimiento sancionador en cuanto resuelva que se ha producido un incumplimiento de la LTPA y tiene encaje dentro de las infracciones previstas. $Y$, en segundo lugar, si es necesario esperar que adquiera firmeza en vía administrativa la resolución del CTPDA de incumplimiento de la LTPA para que se pueda instar la incoación del correspondiente procedimiento, tal y como ha afirmado el propio CTPD en la citada Resolución.

Estas cuestiones las ha dejado resueltas el propio CTPDA en la Resolución PA29/2017, de 13 de septiembre ${ }^{7}$. El mismo Grupo Parlamentario Popular del Parlamento de Andalucía, presentó una denuncia por no haberse publicado en el Portal de Transparencia de la Junta de Andalucia el Anteproyecto de Ley Andaluza de Fomento del Emprendimiento cuando se remitió al Consejo Consultivo de Andalucía dicho anteproyecto para su dictamen. Ello suponía el mismo incumplimiento de las obligaciones de publicidad activa que el caso aquí analizado, pero esta vez el incumplimiento era de la Consejería de Economía y Conocimiento de la Junta de Andalucía. Pues bien, una vez declarado que dicha Consejería incumplió la obligación de publicidad activa establecida en el artículo 13.1.b) de la LTPA durante la tramitación del Anteproyecto de Ley Andaluza de Fomento del Emprendimiento, el CTPD fijó el criterio a seguir cuando se solicite por un denunciante el que inste la incoación este órgano del procedimiento sancionador previsto en el artículo 57 de la LTPA:

"Ante la ausencia de reglamentación aplicable a estos casos, es criterio del Consejo que declarar el incumplimiento de una obligación de publicidad activa y dictar un Acuerdo para instar al órgano a que incoe el procedimiento correspondiente constituyen distintos procedimientos que han de tramitarse y resolverse separadamente, otorgando los pertinentes trámites de alegaciones derivados de la instrucción de ambos procedimientos. En consecuencia, este Consejo procederá a tramitar el procedimiento correspondiente para instar, en su caso, la incoación del procedimiento que corresponda, dando cuenta al ahora denunciante del resultado de los mismos."

7 Resolución número PA-29/2017, de 13 de septiembre, del Consejo de Transparencia y Protección de Datos de Andalucia https://www.ctpdandalucia.es/sites/default/files/denuncias/res-pa-292017.pdf 


\section{El primer expediente sancionador ...}

De este criterio, que pudiera ser cuestionado, se desprende que el CTPDA se reserva la facultad de valorar para cada caso concreto si procede o no instar la incoación del procedimiento previsto en el artículo 57 de la LTPA una vez dictaminado por el propio Consejo el incumplimiento de dicha norma. Dicho criterio podria variarse en posteriores resoluciones y sería interesante, además, comprobar lo que al respecto se vaya a acordar en otras Comunidades Autónomas cuya legislación también contenga un régimen sancionador en la materia.

\section{LA INCOACIÓN DEL EXPEDIENTE SANCIONADOR POR PARTE DE LA CONSEJERÍA DE HACIENDA Y ADMINISTRACIÓN PÚBLICA DE LA JUNTA DE ANDALUCIAA.}

Tras la última Resolución del CTPDA de fecha 13 de junio de 2017 no cabe sino la apertura del expediente sancionador por parte de la Consejeria de Hacienda y Administración Pública. Recordar que la controversia analizada se inició con la denuncia presentada, con fecha 28 de octubre de 2016, por la no publicación en el Portal de Transparencia de la Junta de Andalucía del Anteproyecto de la Ley de Presupuesto de la Comunidad Autónoma de Andalucía para el año 2017.

Pero la tramitación del expediente sancionador queda en la esfera del propio órgano que ha incumplido la LTPA con la única obligación de comunicar el resultado del mismo al CTPDA quedando ajeno el denunciante tanto a la tramitación como a su conclusión final, lo que redunda paradójicamente en una falta de transparencia.

A este respecto señala Pérez Monguió (2015: 318) que "hubiera resultado oportuno que la Ley de Andalucía 3/2005, de 8 de abril o ahora la LTPA, hubiera otorgado a los denunciantes la posibilidad de conocer, al menos, si su acto de denuncia dio lugar a la incoación del procedimiento. Somos conscientes de que la jurisprudencia en materia de régimen disciplinario ha blindado a los empleados "públicos profesionales", bajo el paraguas del mérito profesional y de la capacidad, del interés de terceros pero es muy distinta la condición de las autoridades, que son cargos de confianza política (salvo los electos) y, por tanto, se les debe exigir un plus de transparencia y los asuntos que se deriven de su actuación como cargo público y las infracciones que cometan deberían ser conocidas". Por tanto, añade el autor, "en el caso de denunciantes estimamos que debería ser obligatorio informarles, al menos, de si se procedió a incoar el procedimiento, pues del incumplimiento en los supuestos de infracciones graves y muy graves tendrá conocimiento por medio de los boletines o diarios oficiales". 


\section{Ma Teresa Ruiz-Sillero Bernal}

En este caso concreto y al darse la particularidad de que el denunciante era un grupo político con representación en el Parlamento de Andalucia pudo formularse una pregunta oral en la Comisión de Hacienda y Administración Pública para conocer el resultado del expediente sancionador abierto. En dicha Comisión parlamentaria la Sra. Consejera informó que:

"Se dictó una orden de inicio de actuaciones previas. En dicha orden se designó a una instructora del expediente, a una funcionaria de la Consejería, para que realizara las actuaciones pertinentes con el fin de conocer, como en cualquier expediente sancionador, la realidad de los hechos, la mayor precisión, determinar, en su caso, la responsabilidad administrativa... Y el pasado 25 de enero (de 2018) remitió el informe de conclusiones, acompañado de los diferentes informes que había solicitado en el ejercicio de sus actuaciones. Y el informe, señoría, de la instructora del expediente, concluye que no se aprecia indicios de dolo, de culpa o de negligencia en la actuación de la Consejería de Hacienda en relación con el incumplimiento de la publicidad activa del Anteproyecto de Ley del presupuesto para la consejería del año 2017, declarada por el Consejo de Transparencia y Protección de Datos de Andalucía. En consecuencia, hace apenas unos días se ha dictado orden con el cierre de las actuaciones previas instruidas sin que procedan más actuaciones, al no haberse constatado, como les decia, a juicio de la instructora, la existencia de dolo, culpa o negligencia. Como corresponde, el resultado de la instrucción se ha notificado -o se estará notificando, porque ha sido en estos dias-, al Consejo de Transparencia y Protección de Datos".

Y sobre la cuestión de si constaba en dichas actuaciones previas la identificación del posible infractor o infractores del incumplimiento de la LTPA por no haberse publicado en el Portal de Transparencia el Anteproyecto de la Ley de Presupuesto del año 2017, la Sra. Consejera no aportó ningún detalle al afirmar que no tenía el dato concreto y no podía facilitar ninguna información adicional, aunque reconoció que se había tomado declaración, testimonio, de prácticamente todas las personas que, de alguna manera, intervienen en la tramitación del presupuesto.

Así, el expediente sancionador incoado por el incumplimiento de la obligación de publicidad activa prevista en la LTPA terminó sin fijar ni la posible infracción cometida y sin ninguna propuesta de sanción.

Respecto a la posible infracción cometida y a la sanción aparejada, habría que remitirse al artículo 52 de la LTPA para las infracciones y al artículo 55 de dicho texto legal que establece las sanciones por el incumplimiento de obligaciones de

8 Diario de Sesiones del Parlamento de Andalucía número 479, X Legislatura, 22 de febrero de 2018 , Comisión de Hacienda y Administración Pública, pág. 56. http://www.parlamentodeandalucia.es/webdinamica/portal-webparlamento $/$ pdf.do?tipodoc=diario $\& i d=128114$ 


\section{El primer expediente sancionador ...}

publicidad activa. En este caso concreto denunciado podria tratarse de una infracción leve prevista en la letra a) del artículo 52.3 por el incumplimiento de las obligaciones de publicidad activa previstas en el título II. La sanción correspondiente a dicha infracción sería disciplinaria y si fuera imputable a personal al servicio de las entidades previstas en el artículo 3, se le aplicarian las sanciones que correspondan con arreglo al régimen disciplinario que en cada caso resulte aplicable. En caso de que la infracción fuese imputable a autoridades y directivos correspondería una amonestación por una infracción leve.

\section{CONCLUSIONES.}

1. Resulta acertado que el Consejo de Transparencia tenga la competencia para instar la incoación de un expediente sancionador por incumplimiento de la LTPA.

2. La incorporación de un régimen sancionador en la LTPA ha tenido como consecuencia que no se vuelva a incumplir la LTPA en cuanto a la publicación en el Portal de la Transparencia del Anteproyecto de la Ley de Presupuesto de la Comunidad Autónoma de Andalucía.

3. Las resoluciones del Consejo de Transparencia y Protección de Datos de Andalucía que acuerden la incoación del procedimiento previsto en el artículo 57 de la LTPA podrian publicarse, así como el resultado de la instrucción que se comunique al Consejo.

4.- La decisión del Consejo de Transparencia y Protección de Datos de Andalucía de abrir dos procedimientos diferenciados, uno para declarar el incumplimiento de la norma y otro para instar la incoación, hace que el propio Consejo tenga una mayor discrecionalidad a la hora de tomar la decisión de abrir el segundo.

5. Tendría que ser accesible para el denunciante el contenido del expediente sancionador incoado y deberia el Consejo de Transparencia y Protección de Datos de Andalucia darle traslado del resultado de la instrucción del expediente una vez que el propio Consejo lo haya recibido.

6. El hecho de que la instrucción del expediente sancionador se realice por la propia Administración que ha incumplido la norma resta posibilidades a que termine el mismo en una propuesta de sanción. 


\section{$M^{a}$ Teresa Ruiz-Sillero Bernal}

\section{BIBLIOGRAFÍA.}

Falcón Pliego, J.L. 2018. "La publicidad activa en la Ley 1/2014, de 24 de junio, de Transparencia Pública de Andalucía y en la actividad del Consejo de Transparencia y Protección de Datos de Andalucia", en Troncoso Reigada, A. (Director), Transparencia Pública y Comunidades Autónomas. Valencia: Tirant lo Blanch, 473-509.

Fernández Ramos, S. 2015. "La publicidad activa", en Fernández Ramos, S. (Coordinador), Estudio sobre la Ley de Transparencia Pública de Andalucía. Sevilla: Instituto Andaluz de Administración Pública, 55-194.

García de Enterría, E. y Fernández T.R. 2017. Curso de Derecho Administrativo II. Cizur Menor (Navarra): Aranzadi Civitas.

Pérez Monguió, J.M. 2015. "Régimen sancionador", en Fernández Ramos, S. (Coordinador), Estudio sobre la Ley de Transparencia Pública de Andalucia. Sevilla: Instituto Andaluz de Administración Pública, 291-339.

Rueda Gómez, J. 2015. "Organización de la transparencia: Consejo de Transparencia y Protección de Datos de Andalucia", en Fernández Ramos, S. (Coordinador), Estudio sobre la Ley de Transparencia Pública de Andalucia. Sevilla: Instituto Andaluz de Administración Pública, 263-290. 